Vol. 4, $\mathrm{N}^{\circ} 1$, p.23-42, enero - marzo, 2020

\title{
Plan Estratégico de Marketing para la Empresa Litargmode del Cantón Gualaceo provincia del Azuay - Ecuador
}

\section{Strategic Marketing Plan for the Litargmode Company of the Gualaceo of Azuay - Ecuador}

Kléber Antonio Luna Altamirano. ${ }^{1}$, Johanna Estefanía Rodas Orellana. ${ }^{2}$, Viviana Mercedes Córdova Rodas. ${ }^{3}$, Milton Xavier Uzhca Lata. ${ }^{4}$

DOI: https://doi.org/10.33262/visionariodigital.v4i1.1066

\begin{abstract}
The present article consists of developing a Strategic Marketing Plan for the positioning and increase of participation in the market of the company LITARGMODE, located in the City of Gualaceo, Province of Azuay, considered a tourist canton and especially artisan in the manufacture of footwear. Due to the increasing demand of leather footwear manufacturers, the problem of the present investigation is that the company has notably decreased its sales within the local and regional market due to the entry of foreign products that enter without tariffs and at a lower cost, considering that it is necessary the application of a marketing tool to position and increase the market share of the company in the different sectors of the country. The objective of the study is to design a Strategic Marketing Plan with the purpose of trying to improve sales at a local and regional level. The methodology of the present investigation is of descriptive type with quantitative approach where the deductive method will be applied using the technique of the survey directed to the employees of the Company, suppliers, citizenship and potential clients. The results indicate that
\end{abstract}

${ }^{1}$ Doctor(c) Ciencias Sociales Mención Gerencia, Universidad del Zulia República Bolivariana de Venezuela, profesor investigador Unidad Académica de Administración Universidad Católica de Cuenca, klunaa@ucacue.edu.ec

${ }^{2}$ Estudiante de la carrera de Contabilidad y Auditoría en la Unidad Académica de Administración de la Universidad Católica de Cuenca, johanna.rodas.15@est.ucacue.edu.ec

${ }^{3}$ Estudiante de la carrera de Contabilidad y Auditoría en la Unidad Académica de Administración de la Universidad Católica de Cuenca, viviana.cordova.81@est.ucacue.edu.ec

${ }^{4}$ Estudiante de la carrera de Contabilidad y Auditoría en la Unidad Académica de Administración de la Universidad Católica de Cuenca, milton.uzhca.74@est.ucacue.edu.ec 
there is high competition in the market, advertising for sale is too low and they do not promote the product. With this study and the Strategic Marketing Plan the people in charge will take the best decisions to try to improve this serious problem that hurts the company and thus be a competitive brand in the market.

Keywords: Leather footwear, Strategic Marketing Plan, Sales reduction.

\section{Resumen}

El presente artículo consiste en desarrollar un Plan Estratégico de Marketing para el posicionamiento e incremento de participación en el mercado de la empresa LITARGMODE, ubicada en la Ciudad de Gualaceo, Provincia del Azuay, considerado como un cantón turístico y en especial artesanal en la fabricación de calzado. Debido a la creciente demanda de fabricantes de calzado de cuero, el problema de la presente investigación radica en que la empresa ha disminuido notablemente sus ventas dentro de la plaza local y regional debido a la entrada de los productos extranjeros que entra sin aranceles y a un costo inferior, considerando que se hace necesario la aplicación de una herramienta de mercadeo para posicionar e incrementar la participación de mercado de la empresa en los diferentes sectores del país. El objetivo del estudio es diseñar un Plan Estratégico de Marketing con el propósito de tratar de mejorar las ventas a nivel local y regional. La metodología de la presente investigación es de tipo descriptivo con enfoque cuantitativo en donde se aplicará el método deductivo utilizando la técnica de la encuesta dirigido a los funcionarios de la Empresa, proveedores, ciudadanía y clientes potenciales. Los resultados indican que existe una alta competencia en el mercado, la publicidad para venta es demasiado baja y no promocionan el producto. Con este estudio y a través del Plan Estratégico de Marketing los directivos tomaran las mejores decisiones para mejorar este grave problema que hace daño a la empresa y así ser una marca competitiva en el mercado.

Palabras clave: Calzado de cuero, plan de Marketing Estratégico, reducción en ventas.

\section{Introducción}

La empresa LITARGMODE fundada en el año 1972 en la ciudad de Gualaceo, Provincia del Azuay-Ecuador. Es una de las primeras empresas más grandes dentro del Cantón, que cuenta con una amplia experiencia y reconocimiento a nivel nacional en el mercado, posesionada con su propia marca LITARGMODE, tiene como finalidad brindar satisfacción a sus clientes mayoristas y minoristas con una alta variedad de diseños que ofrece. También cuenta con su propio local ubicada en las calles Av. Jaime Roldós 4-90 y Antonio delgado, siendo la única tienda dentro de la urbe. Generando así la atracción de turistas de otras ciudades y países que viajan hasta la misma para adquirir su calzado a gusto y preferencia del consumidor. Rizo, Vuelta, Vargas y Leyva (2019) afirman: 
El estudio de mercado tiene como finalidad cuantificar el número de individuos, empresas y otras entidades económicas generadoras de una demanda que justifique la puesta en marcha de un determinado programa de producción de bienes o servicios, sus especificaciones y el precio que los consumidores estarían dispuestos a pagar por ellos (p.47).

También explica que las estrategias de comercialización, conocidas como estrategias de mercadeo, consisten en acciones estructuradas y completamente planeadas que se llevan a cabo para alcanzar determinados objetivos relacionados con la mercadotecnia, tales como dar a conocer un nuevo producto, aumentar las ventas o lograr una mayor participación en el mercado (Arechavaleta, 2015).

Así mismo, Ospina y Sanabria. (2010) sostienen:

En lo empresarial (transaccional) se puede entender la gestión de mercadeo como un intercambio con beneficio mutuo, es decir un conjunto de transacciones entre oferentes y demandantes donde se pretende la satisfacción de las necesidades de los consumidores, mediante los productos o servicios adquiridos, mientras que las organizaciones empresariales obtienen los beneficios económicos que ellas legítimamente pretenden. En ese orden de ideas, el éxito de la gestión de mercadeo estaría dado en la generación de relaciones de largo plazo y la fidelidad absoluta de parte de los clientes siendo el área de mercadeo la responsable de la gestión de las actividades que facilitan el intercambio, con beneficio, entre la organización y el cliente (p.110).

Jaramillo (2005) sostiene "Las Pymes son organizaciones económicas que desarrollan actividades industriales, comerciales y de servicios que combinando capital, trabajo y medios productivos obtienen un bien o servicio que se destina a satisfacer diversas necesidades para un sector y un mercado determinado" (p.104). Pérez, García, Sánchez y Campdesuñer (2018) afirman:

La satisfacción de los clientes por lo general no posee niveles mínimos pre establecidos de aceptación, ni límites de tolerancias formalmente planteados más bien debe buscarse y propiciarse un incremento constante de esta, lo que no niega que la misma deba ser medida y que su variabilidad deba ser controlada (p.3). 
ISSN: 2602-8506

Vol. 4, $\mathrm{N}^{\circ} 1$, p.23-42, enero - marzo, 2020

El problema de la presente investigación radica en que la empresa ha disminuido notablemente sus ventas dentro de la plaza local y regional debido a la entrada de los productos extranjeros que ingresan sin aranceles y a un costo inferior. Villegas, Zapata (2007) afirman: "La alta carga impositiva se destaca dentro de los problemas tributarios, la multiplicidad de impuestos y la frecuencia en las reformas tributaria, y en el mercado interno la competencia desleal" (p.26). En los últimos años la competencia ha incrementado de manera muy rápida debido a que existen pequeñas empresas importadoras de materiales de calzado que consiguen materia prima y de acabado a muy bajo precio, lo cual produce una inestabilidad en los costos afectando directamente los precios del mercado. Villegas, Zapata (2007) asevera:

La mayoría de las empresas actualmente no se fortalecen ni expanden debido al temor de una "inundación" del mercado nacional por parte de transnacionales, tal es el caso de las compañías chinas, rescatándose el reconocimiento internacional de la calidad del cuero y en especial del calzado colombiano (p.26).

El objetivo del estudio es diseñar y presentar un Plan Estratégico de Marketing para la empresa LITARGMODE, con el propósito de tratar de mejorar las ventas a nivel local y regional, se puede decir que un Plan Estratégico de Marketing es una herramienta que permite planificar, analizar y tomar decisiones para un correcto funcionamiento de la empresa, que a la vez ayudara a mejorar la situación en la que se encuentra. Por ello, Álvarez, (2007) sostiene:

La estrategia describe cómo se lograrán los objetivos generales, es decir, la dirección futura de los productos y mercados de la organización incluyendo los métodos de intervención que ayudarán a la organización a cumplir su misión y a lograr sus objetivos generales de manera factible y eficaz (p.70).

El mismo autor, Álvarez, (2007) afirma:

La planificación estratégica es la determinación de metas y objetivos a largo plazo de una empresa y la adopción de cursos de acción y asignación de recursos necesarios que permitan la obtención de estas metas. Los planes estratégicos de marketing están basados en la valoración del equipo de planificación de Marketing, percepciones y expectativas de la directiva (p.72). 
En cambio, Bermeo (2014) sostiene:

Planeación estratégica de marketing engloba todas las acciones que tienen que ver con el cumplimiento de los objetivos de marketing (...) Las empresas sean de productos o servicios necesitan contar con un plan estratégico de marketing puesto que para conseguir clientes es necesario decir al público que es lo que se ofrece y dependiendo de cada tipo de negocio y producto se debe actuar de cierta manera, se deben crear estrategias y generar medios por los que se pueda interactuar con el mercado (p.1).

Boso, Cadogan y Story (2007) aseveran:

La planeación estratégica del marketing incluye las herramientas fundamentales para el estudio del entorno externo organizacional, variables asociadas al comportamiento interno del marketing y su relación con los cambios sociales y tecnológicos que acontecen en el sistema empresarial. Sin embargo, todos estos elementos pueden ser analizados, para las pequeñas y medianas empresas, dentro del plan de marketing (p.2).

Este representa un compromiso para mejorar las estrategias de ventas y al mismo tiempo tratar de posicionar un punto en el mercado para la empresa, la presente investigación es de tipo descriptivo, el cual detalla con exactitud el objeto de estudio, no solo recolecta datos si no se estudia y se analiza los resultados más relevantes los cuales constituyen aporte al conocimiento.

En un estudio descriptivo se seleccionan una serie de cuestiones, conceptos o variables y se mide cada una de ellas independientemente de las otras, con el fin, precisamente, de describirlas. Estos estudios buscan especificar las propiedades importantes de personas, grupos, comunidades o cualquier otro fenómeno (Cazau, 2006, p.27).

El presente estudio, tiene su enfoque desde lo cuantitativo, con el fin de tratar de mejorar este grave problema que hace daño a la empresa y así ser una marca competitiva en el mercado. Enfoque cuantitativo, Utiliza la recolección de datos para probar hipótesis con base en la medición numérica y el análisis estadístico, con el fin establecer pautas de comportamiento y probar teorías (Hernández, Fernández y Baptista, 2014, p.4).

El método que se aplicará será el deductivo, ya que este se refiere a una forma del razonamiento o pensamiento, parte de lo general para llegar a algo especifico. Método deductivo es una forma de 
razonamiento que parte de una verdad universal para obtener conclusiones particulares (Maya, 2014, p.14).

El estudio se estructura, indicando en primera instancia la revisión del estado del arte, en el cual se soporta teóricamente la investigación, partiendo de la revisión de investigaciones sobre el tema en mención como parte de la metodología se detalla la elaboración del modelo de un Plan Estratégico de Marketing para la empresa LITARGMODE. Posteriormente, se plantea los estados orientados a la incrementación en ventas de la empresa, posicionando a la compañía en el mercado, mejorar sus ventas y por ende contribuirá al desarrollo del cantón que se caracteriza por la confección de calzado de cuero, como conclusión se logrará obtener un plan estratégico de marketing que sea eficiente y eficaz, con ello se generará una mayor productividad en ventas para lograr resultados óptimos.

\section{Estado del arte}

El diseñar un Plan Estratégico de Marketing para la empresa LITARGMODE del cantón Gualaceo, provincia del Azuay Ecuador, es de suma importancia ya que este permitirá el incremento y su posicionamiento en el mercado a nivel nacional, por ello algunos autores contribuyen con sus investigaciones relacionados al tema de estudio entre ellos: Chung (2009) Se enfoca en las diferentes técnicas de análisis de escenarios a largo plazo, que responde a la siguiente pregunta cómo concatenar la formulación de los diversos planes estratégicos de una organización hacia un futuro deseado en el largo plazo?: se analizan y compara tres herramientas principales como el Forecasting, el Foresight y la Prospectiva Estratégica, proponiendo con ello un esquema de “Escalera Estratégica”. Rizo, Vuelta, Vargas y Leyva (2019) diseñan y aplican una estrategia de comercialización que permitió brindar servicios de excelencia tanto en atención al cliente y comercialización de productos cárnicos porcinos, alcanzando a mejorar el posicionamiento en el mercado de la Ciudad de Santiago de Cuba, y logra cambiar la mentalidad para la toma de decisiones acertadas acerca de cómo se puede mejorar la venta de los productos cárnicos. Rizo, Vuelta, Vargas y Leyva (2019) con el propósito de exponer estrategias de comercialización para la gestión de ventas en el mercado, realizan un análisis detallado de cada actividad, con su respectiva valoración y comparación de precios aprobados de venta, evalúan cada entorno para la propuesta de un conjunto de conclusiones y recomendaciones que contribuyen a que la dirección posea más 
elementos para transformar dicha situación, y se centran en diferentes estudios de mercado, aplicando la encuesta a 286 personas que realizaron compras en los mercados agropecuario. Por su parte, los autores Idrovo, Luna, Ordoñez y Lituma (2019) presentan la siguiente investigación donde proponen plantear un plan de marketing estratégico para la promoción y difusión del turismo en el Municipio de Nabón el cual permitirá una mejor compresión para establecer las bases y estrategias encaminadas para alcanzar un desarrollo turístico que sea sostenible y competitivo, aplican un instrumento de recolección de datos a 150 informantes de la zona, los resultados indican que se debe capacitar al talento humano perteneciente al GAD Municipal del cantón Nabón para las actividades que deben realizarse en beneficio del desarrollo turístico en la zona. A su vez Castro, Mercado, Londoño y Hoyos (2017) proponen elaborar un plan de marketing que este inspirado en la investigación formal de mercados, para identificar aquellos insigths de valor, que facilitaran una mayor fluidez en la comunicación con las diferentes plazas de mercado y así lograr mejorar el reconocimiento en lo que piensa el consumidor final.

Por ello Mogrovejo, Luna, Ormaza, Castro y Torres proponen un plan de marketing que permitirán mejorar la rentabilidad de las empresas del sector de salud que fueron mencionados, en las áreas: imágenes diagnósticas, laboratorios, hospitalización, unidades de cuidados intensivos adultos, pediátrica y neonatal, y farmacia, aplican el método de entrevista y cuestionarios donde permiten analizar información para establecer diferentes propuestas, donde definen diferentes estrategias de marketing para mejorar los productos y servicios que ellos ofrecen para mejorar su rentabilidad.

Los autores aportan un correcto desarrollo de la construcción de un modelo de plan de marketing, ya que tiene una relación directa con el proyecto táctico de la empresa de calzado, con ello se busca mejorar el nexo e interdependencia, así propenderá a optimizar la gestión empresarial dando lugar a mejoras continuas y con ello llevar a cabo el diseñar un Plan Estratégico de Marketing con el propósito de tratar de mejorar las ventas a nivel local y regional de la empresa LITARGMODE.

\section{Metodología}

La presente investigación es de tipo descriptivo con enfoque cuantitativo por lo que se aplica el método deductivo para el diseño de un modelo de un Plan Estratégico de Marketing, la información es suministrada a través de una encuesta dirigida a 40 funcionarios de la empresa LITARGMODE, ubicada en la Ciudad de Gualaceo, Provincia del Azuay, quienes conforman el universo total de 
ISSN: 2602-8506

Vol. 4, $\mathrm{N}^{\circ} 1$, p.23-42, enero - marzo, 2020

trabajadores, con el objetivo de dar mayor veracidad a esta información. A continuación, se detallan los cargos del personal a quienes estará dirigida la encuesta.

Tabla 1. Segmentación de empleados de la Empresa LITARGMODE

Fuente: Elaboración propia

\begin{tabular}{lr}
\hline Empleados & Número \\
\hline Gerente & 1 \\
Secretaria & 1 \\
Recepcionista & 1 \\
Jefe operativo & 1 \\
Contador & 1 \\
Choferes & 2 \\
Despachador de mercaderías & 1 \\
Empleados & 31 \\
\hline Total empleados & $\mathbf{3 9}$
\end{tabular}

\section{Resultados}

Como se puede evidenciar en la tabla 1, la población de los trabajadores de la empresa LITARGMODE ubicada en la Ciudad de Gualaceo, Provincia del Azuay, son de 40 empleados, a los cuales se realizó el estudio mediante una encuesta. Se presenta las preguntas más relevantes realizadas en las encuestas con el propósito de estructurar este plan.

Figura 1. Equipo de oficina

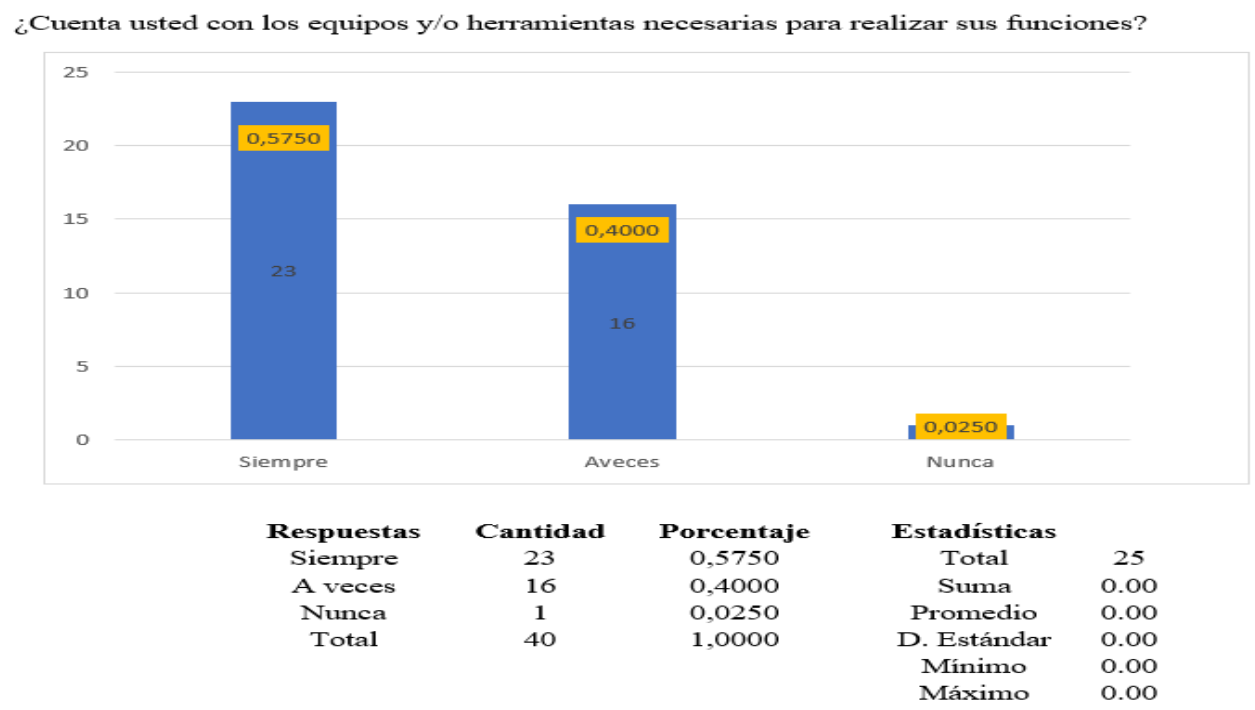

Fuente: Elaboración propia 
ISSN: 2602-8506

Con respecto a la dotación de equipos de oficina, el 57\% de los funcionarios expresan que siempre y el $40 \%$ expresan que a veces tienen lo necesario para desarrollar sus funciones de manera adecuada. Todo colaborador que labora en una oficina debe contar con buenos equipos y mobiliarios para un mejor desempeño. La maquinaria y equipos de oficina comprenden todos los artículos necesarios para desarrollar con eficiencia los procesos productivos o prestación de servicios, su selección debe realizarse de acuerdo con las características de cada puesto de trabajo (Córdova, 2011).

Figura 2. Método de ventas

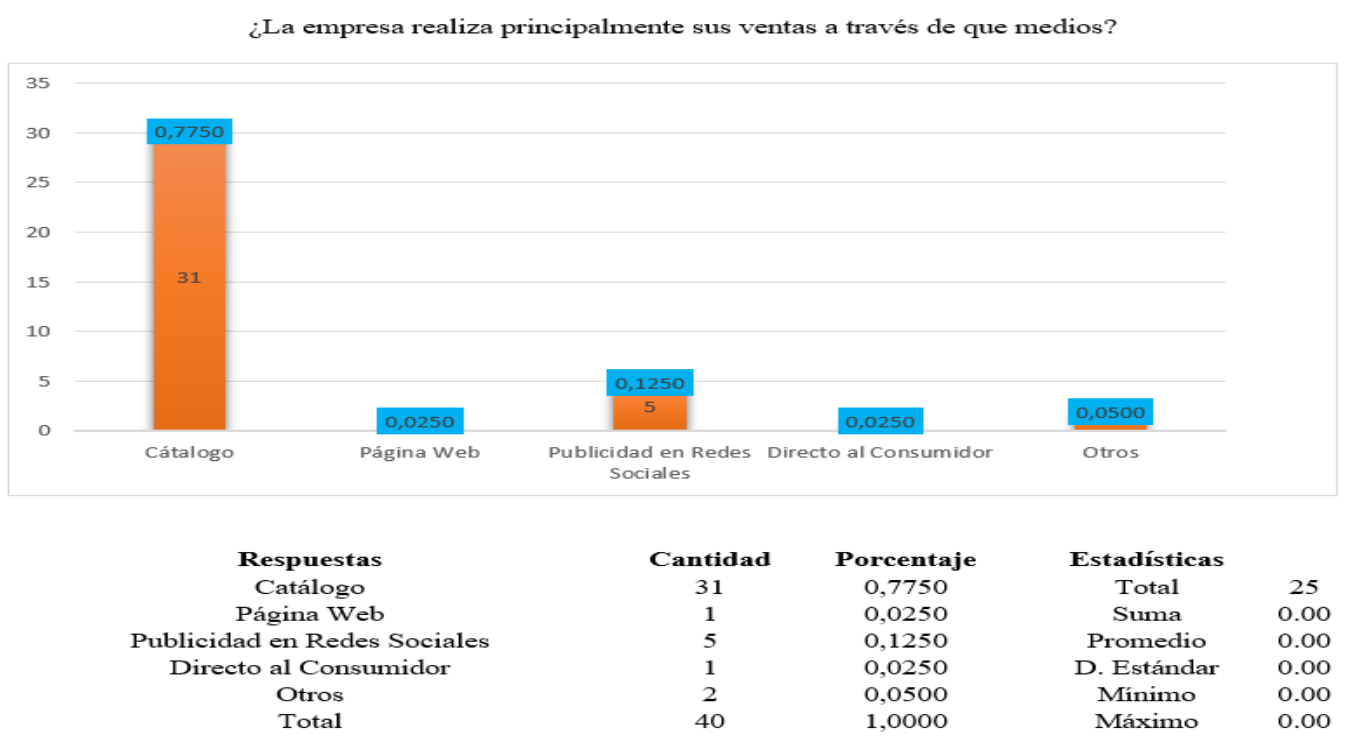

Fuente: Elaboración Propia

En el grafico 2 con respecto al método de ventas el 77\% de los empleados que forman la empresa respondieron que siempre se la realiza por catálogo, y no utilizan otros medios. La venta por catálogo es una muy buena opción para exponer la mercadería, pero también se puede utilizar los medios tecnológicos como, páginas web, publicidad en redes sociales para promocionar sus productos, de tal forma, que los posibles compradores puedan conocer los beneficios a través de ese sitio web, en el caso de que estén interesados y puedan efectuar la compra online. Arce (2011) afirma: "Añadir productos a una línea que ya tiene éxito funciona si se amplía el mercado estimulando o cubriendo distintas necesidades de los clientes, o si la competencia ya ha realizado con éxito la ampliación" (p.93). 
Figura 3. Disminución de ventas

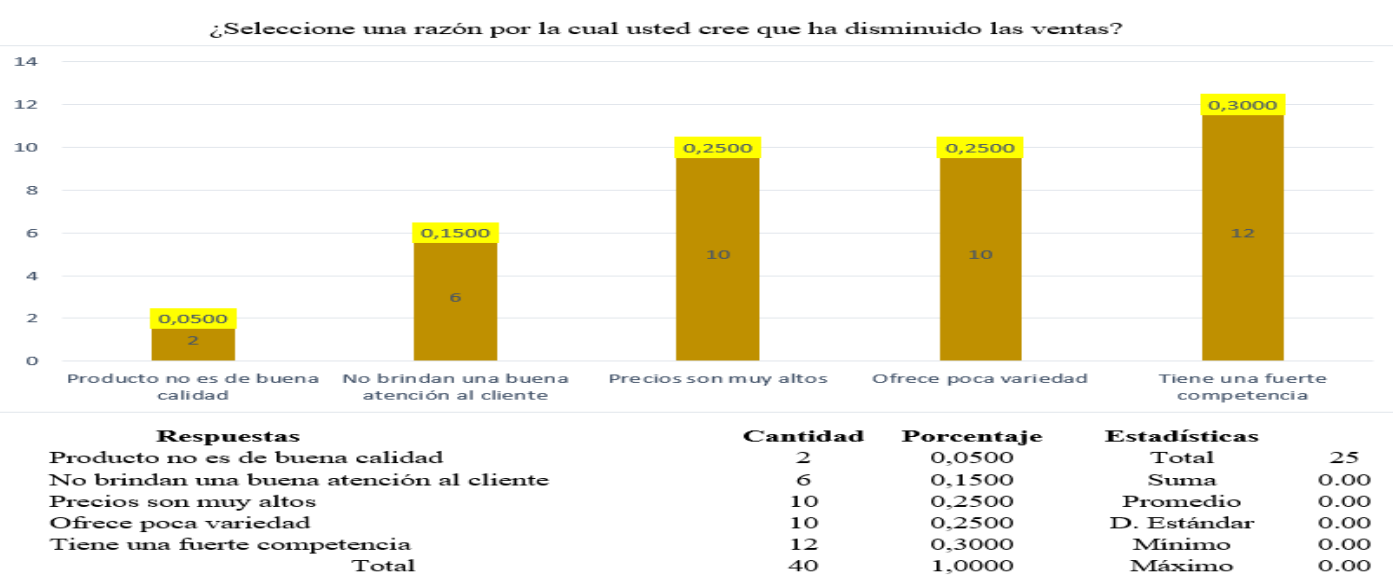

Fuente: Elaboración Propia

En el grafico 3 con respecto a la disminución en ventas el 30\% de los empleados respondieron que existe una fuerte competencia de mercado, lo que indica la disminución de ventas, hay una significativa demanda entre empresas que participan en un mercado y que aplican mejores estrategias de manera que pueden minimizar sus costos, maximizar sus ganancias y así mantenerse activas e innovadoras frente a otras empresas. León (2013) asevera:

Considerando la competitividad como una capacidad de las empresas que comienza desde si hacia la vinculación con el exterior logrando eficiencia interna de los procesos, productos y personal, es la fuerza de ventas la parte de este personal que se proyecta al entorno, siendo generadores de ingresos monetarios a partir de donde se consiguen las ganancias y rentabilidad, indispensables para perdurar en el tiempo (p.387)

Figura 4. Calidad el Producto

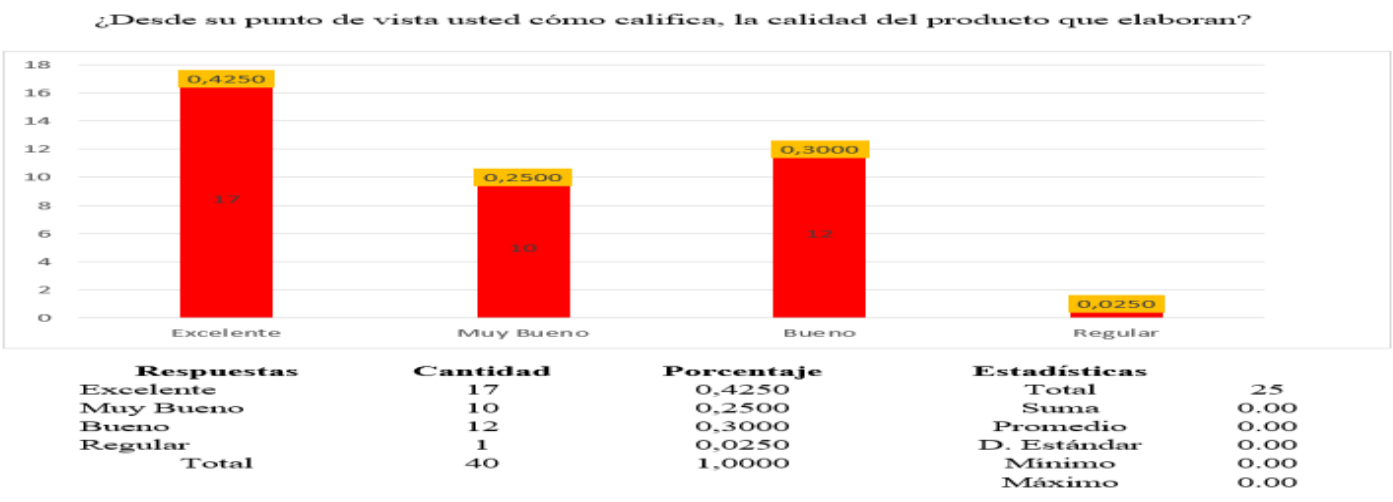

Fuente: Elaboración propia 
Vol. 4, $\mathrm{N}^{\circ} 1$, p.23-42, enero - marzo, 2020

En el grafico 4, con respecto a la calidad del producto el $42.5 \%$ de los empleados respondieron que es excelente. La calidad de producto es la precepción que el cliente tiene del mismo, es una fijación mental del consumidor que asume conformidad con dicho producto o material y la capacidad del mismo para satisfacer sus necesidades. López (2005) asevera:

Una definición generalmente aceptada es la que identifica la Calidad con el grado de satisfacción que ofrecen las características del producto/servicio, en relación con las exigencias del consumidor al que se destina, es decir, un producto o servicio es de calidad, cuando satisface las necesidades y expectativas del cliente o usuario, en función de determinados parámetros, tales como seguridad, confiabilidad y servicio prestado (p.4).

Figura 5. Posicionamiento del Producto

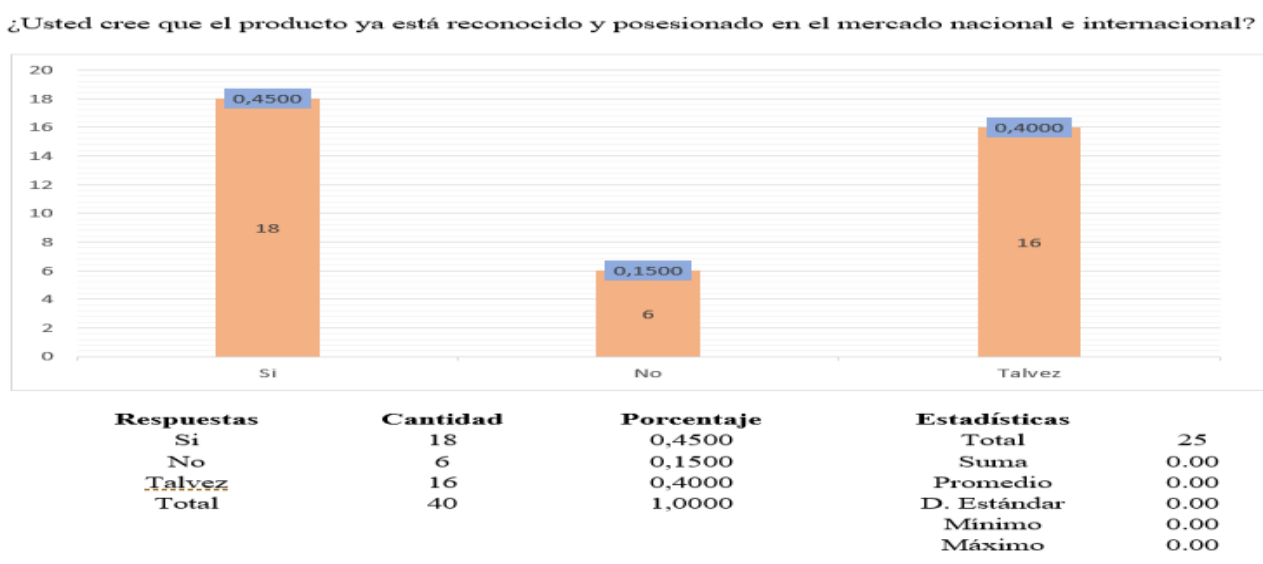

Fuente: Elaboración Propia

Con relación al posicionamiento del producto, el $45 \%$ de los empleados respondieron que sí. El posicionamiento de una empresa permite que una marca destaque sobre las demás en todo el ámbito en donde haya presencia de muchos productos similares que ofrezcan características parecidas. Ortegón (2017) afirma:

La imagen y posicionamiento de marca es un proceso complejo que demanda procedimientos de análisis pertinente debido a la proliferación de nociones y su impacto sobre la estrategia empresarial. Poder indagar las características y atributos percibidos por el mercado brinda información valiosa a las compañías para facilitar sus decisiones en actividades e inversiones en marketing (p.26). 
Figura 6. Promoción del Producto

Vol. 4, $\mathrm{N}^{\circ} 1 .$, p.23-42, enero - marzo, 2020

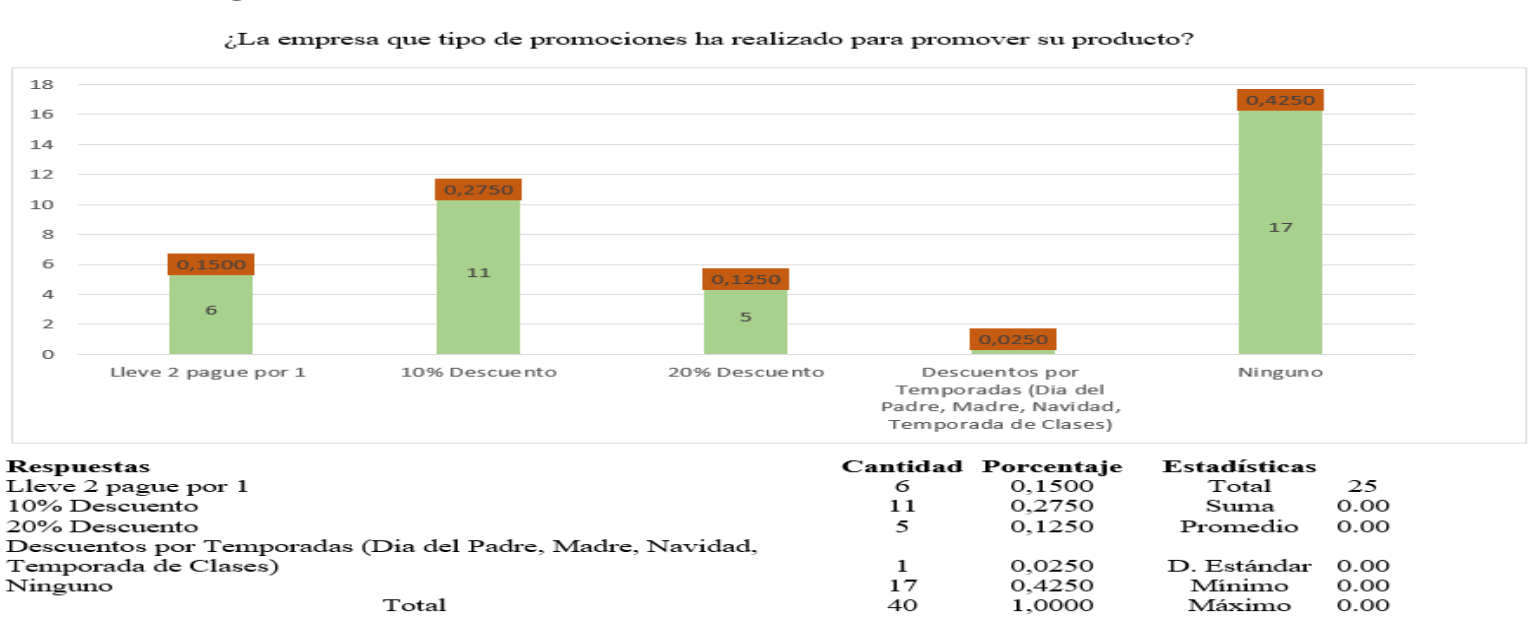

Fuente: Elaboración Propia

Con respecto a la Promoción del Producto el $42.5 \%$ de los empleados respondieron que no existe ninguna. En una empresa la promoción radica en comunicar, dar a conocer la existencia de mercadería o producto a los consumidores. En la promoción, los vendedores pretenden persuadir, convencer e inducir al cliente a la compra de los productos y así obtener ganancias significativas. Villalba (2005) afirma:

La promoción del producto consiste en comunicar, informar y dar a conocer o presentar la existencia de un producto o servicio a los consumidores. A través de la promoción, los vendedores pretenden persuadir, convencer e inducir al cliente a la compra de sus productos (p.210). $\quad$ Figura7. Oportunidad de mercado

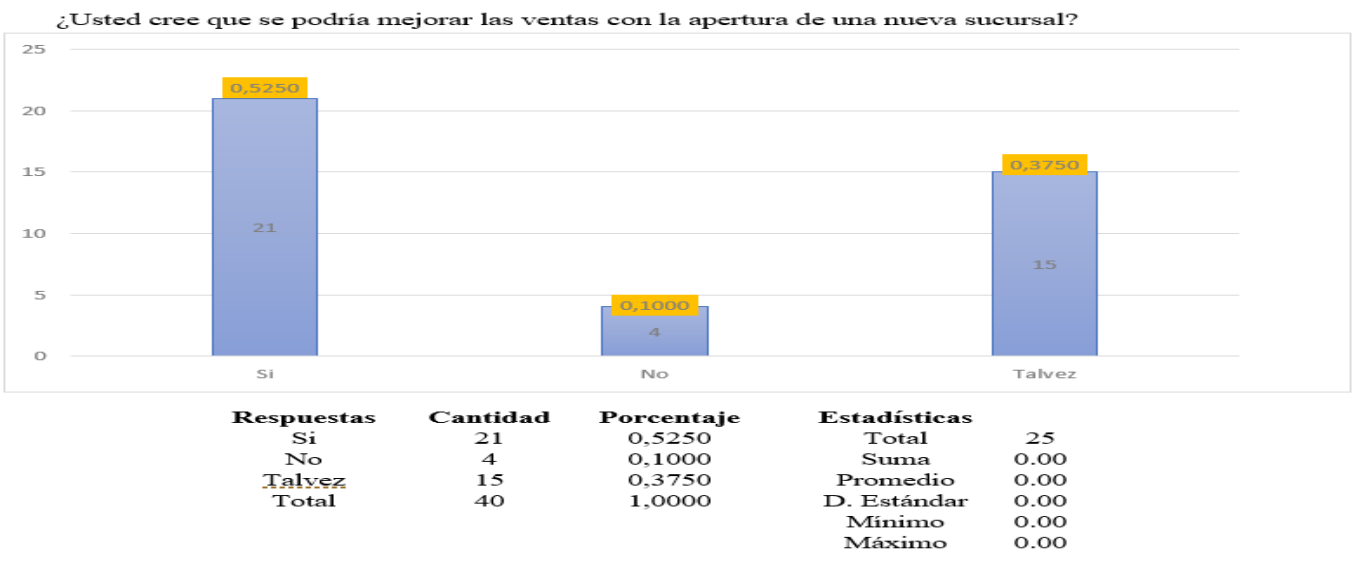

Fuente: Elaboración Propia 
ISSN: 2602-8506

Vol. 4, $\mathrm{N}^{\circ} 1 .$, p.23-42, enero - marzo, 2020

En el grafico 7, con respecto a la Oportunidad de Mercado el 52.50\% de los empleados respondieron que se podría mejorar las ventas con la apertura de una nueva sucursal ya que así se exhibirían más los productos de calzado y obtendrían nuevos clientes e incrementarían sus ventas y ganancias. Singh (2001) afirma:

Esta forma de entender la oportunidad emprendedora exige que ésta genere rentabilidad para ser considerada como tal, por lo que limita las posibilidades de investigación empírica. El autor considera que el análisis de las oportunidades debe llevarse a cabo desde los primeros estadios del proceso de reconocimiento de la misma y antes de que el nuevo negocio sea creado y genere beneficios (p.112).

Figura 8. Publicidad del Producto

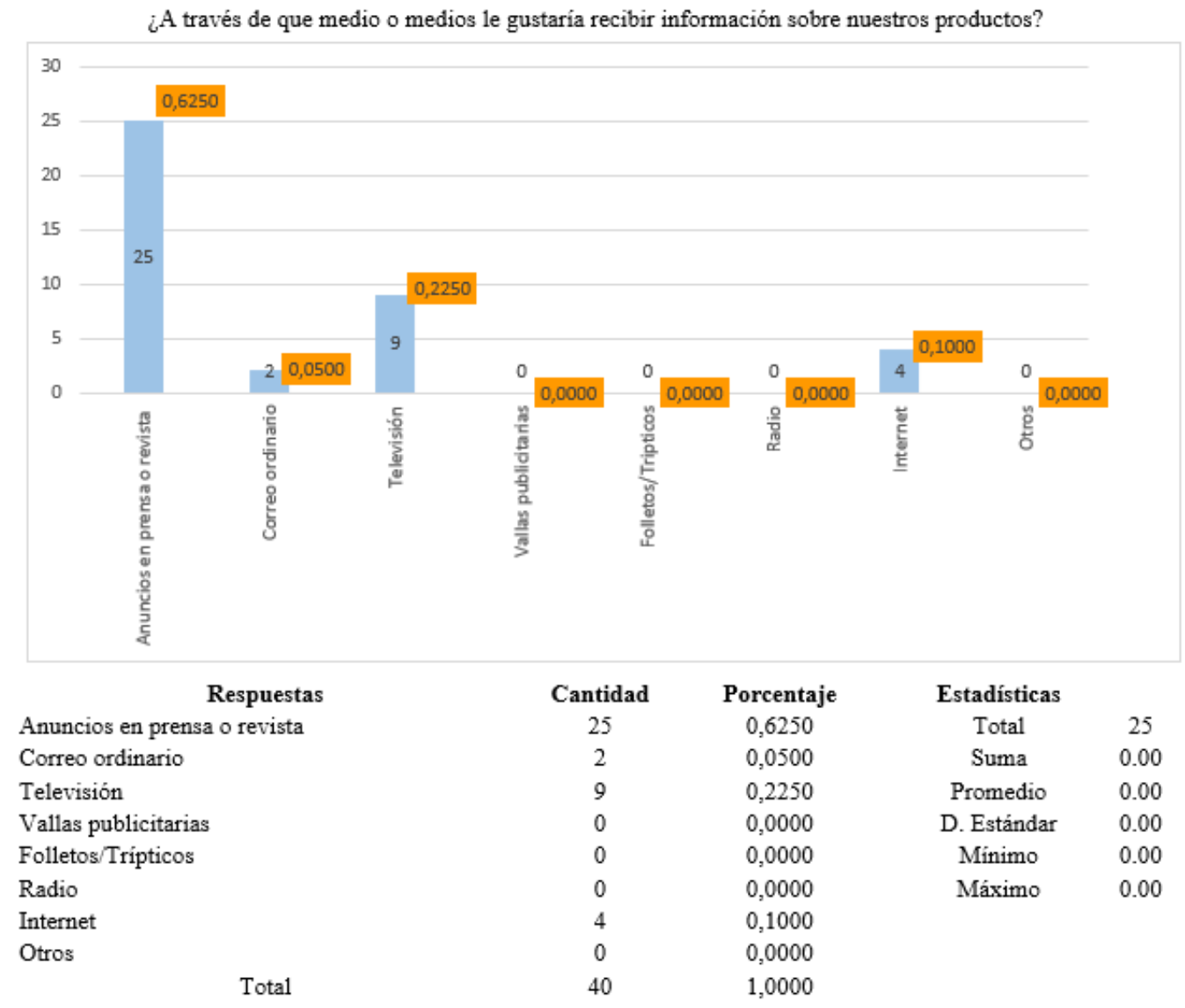

Fuente: Elaboración Propia 
ISSN: 2602-8506

Vol. 4, $\mathrm{N}^{\circ} 1 .$, p.23-42, enero - marzo, 2020

Con respecto a la Publicidad del producto, el $62.50 \%$ de los empleados respondieron que les gustaría recibir información acerca de los productos mediante anuncios en prensa o revista. Un producto es el conjunto de características que engloba la forma, tamaño, color entre otras que busca conseguir una mejor penetración en el mercado y ser alta mente competitivo buscando satisfacer las necesidades del comprador. Martínez (2016) sostiene:

La publicidad es considerada una técnica de comunicación destinada a difundir masivamente mensajes para persuadir a la audiencia al consumo, como una forma de dar a conocer lo que ofrece el negocio, a través de medios gráficos, diarios, revistas, radio, internet, redes sociales, páginas amarillas, televisión incluso en el cine; aunque la mejor publicidad es expandirse en boca a boca de los clientes en forma gratuita, para esto se recomienda tener página webs o blog y emplear Twitter, Instagram, Facebook (p.54).

Para el diseño del Plan Estratégico de Marketing, es necesario estructurar la matriz FODA, este de elabora en base a la información suministrada en las encuestas realizadas a los 40 funcionarios de la empresa, en esta se analiza las fortalezas, oportunidades, debilidades y amenazas de la empresa de calzado. A continuación, en la tabla 2, se indican factores internos.

Tabla 2. Matriz FODA Factores Internos

\begin{tabular}{ll}
\hline \multicolumn{1}{c}{ Fortalezas } & \multicolumn{1}{c}{ Debilidades } \\
\hline Producto, calidad y confiabilidad & Diseños no acordes a la moda juvenil \\
Personal experto & Reducida publicidad \\
Facilidad de entrega directa & Poca capacitación \\
\hline
\end{tabular}

Fuente: Elaboración Propia

Una vez realizada la matriz FODA, en la variable tanto interna como externa que son las que influyen de manera directa con el crecimiento de la empresa y el desempeño de sus trabajadores, se requiere evaluar paso a paso la situación en la que se encuentra la empresa internamente además se determina las fortalezas y debilidades más relevantes de la empresa de calzado, se observa directamente la situación actual de la empresa en estudio, cada una de las variables ayudara a tomar decisiones estratégicas que será el nexo que permita ver la realidad del problema de reducción en ventas. Posteriormente, al desplegar los factores externos (oportunidades y amenazas), se tiene la tabla 3. 
ISSN: 2602-8506

Vol. 4, $\mathrm{N}^{\circ} 1$, p.23-42, enero - marzo, 2020

Tabla 3. Matriz FODA Factores Externos

\begin{tabular}{ll}
\hline \multicolumn{1}{c}{ Oportunidades } & \multicolumn{1}{c}{ Amenazas } \\
\hline Productos similares de baja calidad & Mercado este abastecido \\
Exportación a países vecinos & Situación económica del país \\
Alta demanda de mercado & Ingreso de mercadería ilícita \\
\hline
\end{tabular}

Fuente: Elaboración Propia

La matriz FODA permite establecer una correlación, así se puede visualizar la situación actual de la empresa de calzado para tener un correcto diagnostico para la toma decisiones adecuadas hacia la investigación, permite definir y formular un contexto que permita caracterizar la problemática, definición y formulación que explique los lineamentos a seguir. Se presenta el Modelo del Plan Estratégico de Marketing, en base al análisis de la información más importante adquirida a través de las encuestas, la matriz FODA, fue importante para la estructura de este modelo, con ello se pretende generar cambios a nivel de la empresa LITARGMODE del cantón Gualaceo, provincia del Azuay-Ecuador.

Tabla 4. Modelo de Plan Estratégico de Marketing

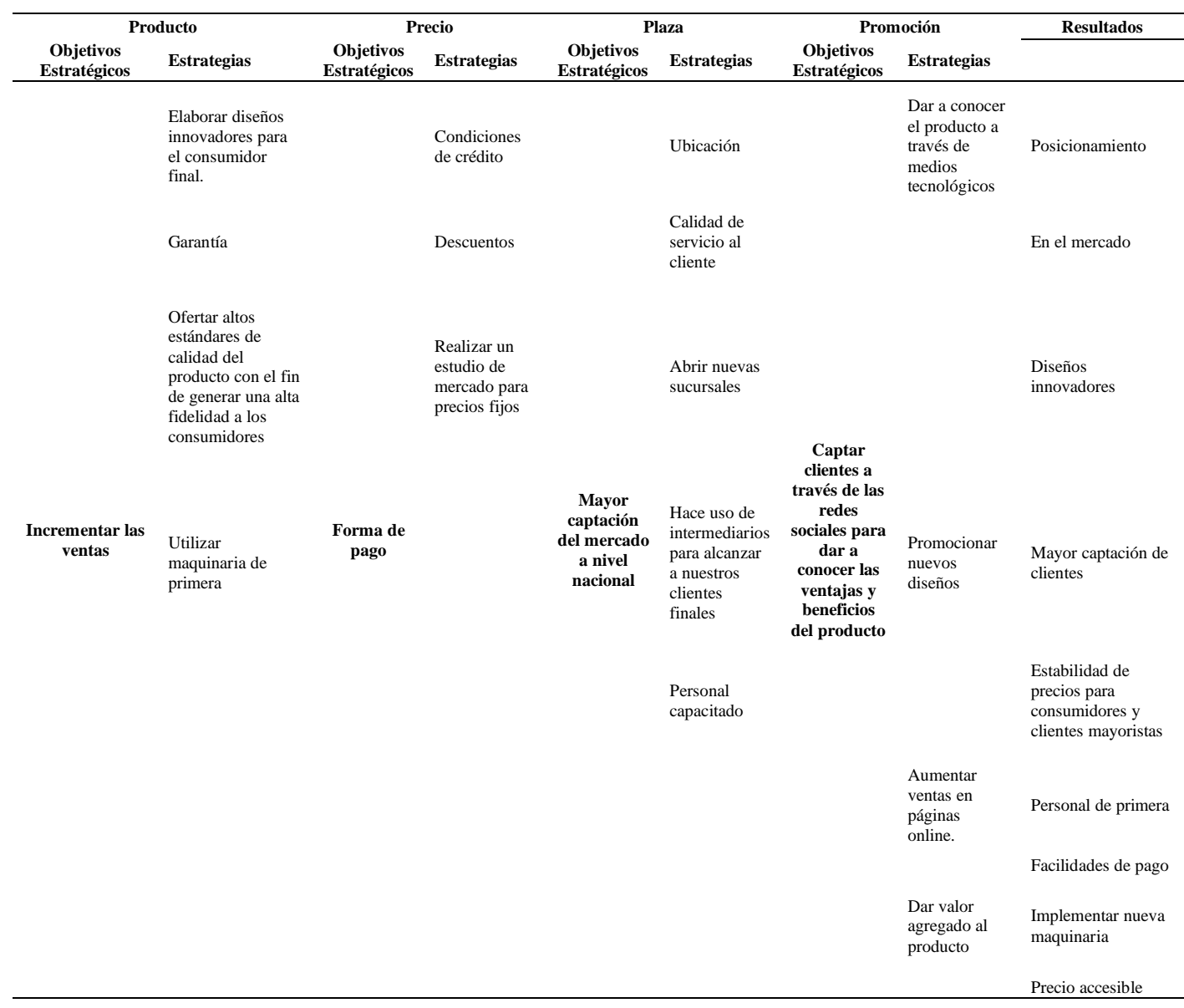


ISSN: 2602-8506

Vol. 4, $\mathrm{N}^{\circ} 1 .$, p.23-42, enero - marzo, 2020

En la tabla 4, se observa los cambios que debe realizar la empresa de calzado. Este modelo de Plan Estratégico de Marketing aplica el uso de las 4P donde se analiza (producto, precio, plaza y promoción), porque se considera que ya han cumplido su ciclo y que están obsoletas para las condiciones del mercado actual, con la finalidad de incrementar las ventas, y con ello lograr un mejor posicionamiento de la marca promocionando su producto de calzado y mercadería mediante redes sociales. Con la aplicación de este modelo, la empresa mejoraría su gestión empresarial a través de:

* Capacitar al personal en todas las áreas, potenciando los valores de profesionalismo y calidad de servicio.

* Permitirá incrementar el nivel de aceptación de los consumidores, atraer a nuevos clientes con una nueva oferta de precios.

* Publicidad agresiva a través de la utilización de medios de comunicación a nivel local y regional.

* Realizar un seguimiento de estrategias mediante los métodos de control propuesto para su efectiva funcionalidad.

* Proteger el mercado en cuanto al precio que se brinda al cliente mayorista y al cliente final.

* Debemos considerar la presencia de productos innovadores, con diseños especiales de calidad que garantiza la satisfacción de los clientes.

* Crear nuevas asociaciones con los mejores clientes y proveedores del mercado, proporcionando un valor agregado a los productos que comercializamos.

\section{Conclusiones}

El diseñar un Plan Estratégico de Marketing, para la empresa LITARGMODE del cantón Gualaceo, provincia del Azuay-Ecuador, tiene el propósito de mejorar las ventas a nivel local y regional de acuerdo con las necesidades exigentes que requiere la empresa, sus clientes y la sociedad en general. La propuesta presentada en este estudio da cumplimiento al objetivo planteado y se orienta en gestionar de una manera más apropiada las 4P, mediante el diseño de las estrategias para cada componente que conforma el modelo, con ello la empresa de calzado podrá incrementar sus ventas y posicionamiento en el mercado.

Este modelo representa una alternativa para el desarrollo del Plan Estratégico de Marketing, que posibilita no solo el mejoramiento de la empresa, será la base fundamental para captar a nuevos y 
ISSN: 2602-8506

Vol. 4, $\mathrm{N}^{\circ} 1 .$, p.23-42, enero - marzo, 2020

posibles clientes e impulsar un importante crecimiento de la empresa, estos factores se analizaron para dar mayor fortalecimiento, tratando de tener una mayor aceptación y posicionamiento en el mercado local y regional.

El modelo de Plan Estratégico de Marketing que se propone se enmarca en las necesidades de la empresa y clientes, mediante tácticas que permitan potenciar su recurso dentro del mercado, convirtiéndose en una empresa potencial, este permitirá generar ventajas competitivas para la misma, con ello se espera adquirir mayores recursos económicos para poder llegar al éxito empresarial.

El presente Plan Estratégico de Marketing ayudará a optimizar los recursos dando un impacto positivo mediante la eficiencia y eficacia, el cual permitirá mejorar los procesos y con ello la rentabilidad de la empresa.

\section{Referencias Bibliográficas}

Álvarez, F. (2007). Planificación estratégica de marketing perspectivas. Perspectivas, 1(20), 67104.https://laclassedotblog.files.wordpress.com/2018/06/425942331006.pdf

Arboleda, H. (2016). Competitividad: Concepto y Evolución Histórica. Revista de Economía \& Administración, 13(2), 14-28. https://www.uao.edu.co/sites/default/files/revista\%20articulo\%201_0.pdf

Arechavaleta Vázquez, E. F. (2015). Estrategias de comercialización. En Ramírez-Ortiz, M. E. (Ed.). Tendencias de Innovación en la Ingeniería de Alimentos (pp. 169-195). Barcelona, España: OmniaScience.

Boso, N., Cadogan, J., y Story, V. (2012). Entrepreneurial orientation and market orientation as drivers of product innovation success: A study of exporters from a developing economy. International Small Business Journal 31 (1), 57-81. DOI: 10.1177/0266242611400469

Castro, A., Mercado, L., Londoño, M., y Hoyos, A. (2017). Plan de marketing para el posicionamiento regional universitario: Caso Universidad Pontificia Bolivariana seccional Palmira. Saber, Ciencia y Libertad, 12(2), 233-247. Recuperado de https://revistas.unilibre.edu.co/index.php/saber/article/view/1592/1176

Cazau, P. (2006). Introducción a la Investigación en Ciencias Sociales. Buenos aires: tercera edición. 
https://www.academia.edu/8000535/Pablo_Cazau_INTRODUCCI\%C3\%93N_A_LA_IN VESTIGACI\%C3\%93N_EN_CIENCIAS_SOCIALES

Chun, A. (2009). Prospectiva estratégica: más allá del plan estratégico. Industrial Data, 12(2), 27 31. Recuperado de https://www.redalyc.org/articulo.oa?id=81620150004

Davis, S. M. (2000). Brand Asset Management. Driving Profi table Growth Trough Your Brands, Jossey-Bass Inc, San Francisco.

Hernández, R. Fernández, C. y Baptista, M. (2014). Metodología de la Investigación. México: sexta edición. MCGRAW-HILL. https://www.uv.mx/personal/cbustamante/files/2011/06/Metodologia-de-laInvestigaci\%C3\%83\%C2\%B3n_Sampieri.pdf

Idrovo Mogrovejo, E., Luna Altamirano, K., Ordoñez Parra, J., \& Lituma Yascaribay, M. (2019). Marketing estratégico turístico para la Provincia del Azuay-Ecuador. Visionario Digital, 3(2), 191-212. Recuperado de https://doi.org/10.33262/visionariodigital.v3i2.408

Jaramillo, O. (2005). Gestión del talento humano en la micro, pequeña y mediana empresa vinculada al programa Expopyme de la Universidad del Norte en los sectores de confecciones y alimentos. Pensamiento \& Gestión, 1(18), 103-137. Recuperado de https://mafiadoc.com/redalycgestion-del-talento-humano-en-la-micro-pequea-y_5a1f153d1723dd8341a70c4b.html

León, N. (2013). Fuerza de ventas determinante de la competitividad empresarial. Revista de Ciencias Sociales, 19(2), 379-389.https://www.redalyc.org/pdf/280/28026992014.pdf

López, R. (2005). La calidad total en la empresa moderna. Perspectivas, 8(2), 67-81. Recuperado de https://www.redalyc.org/pdf/4259/425942412006.pdf

Martínez, D. (2016). Factores clave en marketing enfoque: empresas de servicios. Orbis. Revista Científica Ciencias Humanas, 12(24), 42-58. https://www.redalyc.org/pdf/709/70946593003.pdf

Maya, E. (2014). Métodos y Técnicas de Investigación (primera edición electrónica). México. https://arquitectura.unam.mx/uploads/8/1/1/0/8110907/metodos_y_tecnicas. pdf 
ISSN: 2602-8506

Vol. 4, $\mathrm{N}^{\circ} 1$., p.23-42, enero - marzo, 2020

Mogrovejo Lazo, A., Luna Altamirano, K., Ormaza Andrade, J., Castro Vazquez, P., \& Torres Beltrán, A. (2019). Plan de marketing en el sector salud. Ciudad de Cuenca, Ecuador. Ciencia Digital, 3(2.3), 5-22. https://doi.org/10.33262/cienciadigital.v3i2.3.483

Ortegón, L. (2017). Imagen y posicionamiento de marcas en la industria de agroquímicos en Colombia. Revista Lasallista de Investigación, 14(1), 13-28. Recuperado de https://www.redalyc.org/pdf/695/69551301002.pdf

Ospina, M., y Sanabria, P. (2010). Un enfoque de mercadeo de servicios educativos para la gestión de las organizaciones de educación superior en Colombia: el modelo migme. Revista Facultad De Ciencias Económicas, 18(2), 107-136. Recuperado de https://doi.org/10.18359/rfce.2275

Pérez, R., García, G., Sánchez, A. y Campdesuñer, I. (2018). La satisfacción de clientes en el turístico: metodología seis sigmas. Ciencias Holguín, 24(1), 1-11. Recuperado de https://www.redalyc.org/articulo.oa?id=181553863004

Rizo, M., Vuelta, D., Vargas, B. y Leyva, E. (2019). Estrategia de comercialización para mejorar la gestión de ventas en la empresa porcino Santiago de cuba. Ciencia en su PC,1(4), 91-102. Recuperado de https://www.redalyc.org/articulo.oa?id=181353794007

SINGH, R.P. (2001): "A comment on developing the field of entrepreneurship through the study of opportunity recognition and exploitation”, Academy of Management Review, Vol. 26, No. 1, pp. 10-12.

Villalba, F. (2005). La promoción de ventas y los beneficios percibidos por el consumidor. Revista europea de dirección y economía de la empresa, 14(3), 209-222. Recuperado de https://dialnet.unirioja.es/servlet/articulo?codigo=1399311

Villegas, D. y Zapata, H. (2007). Competitividad sectorial internacional Caso: sector del cuero y del calzado. Entramado, 3(1), https://www.redalyc.org/articulo.oa?id=265420385004

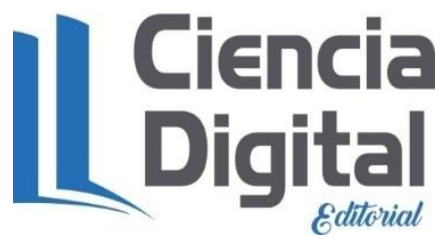


ISSN: 2602-8506

Vol. 4, $\mathrm{N}^{\circ} 1 .$, p.23-42, enero - marzo, 2020

\section{PARA CITAR EL ARTÍCULO INDEXADO}

Luna Altamirano, K. A., Rodas Orellana, J. E., Córdova Rodas, V. M., \& Uzhca Lata, M. X. (2020). Plan Estratégico de Marketing para la Empresa Litargmode del Cantón Gualaceo provincia del Azuay - $\quad$ Ecuador. Visionario Digital, $4(1), \quad$ 23-42. https://doi.org/10.33262/visionariodigital.v4i1.1066

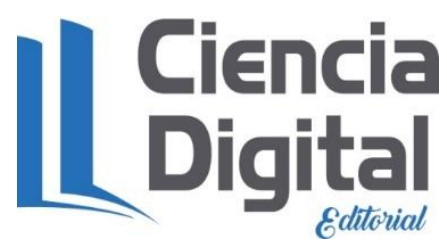

El artículo que se publica es de exclusiva responsabilidad de los autores y no necesariamente reflejan el pensamiento de la Revista Visionario Digital.

El artículo queda en propiedad de la revista y, por tanto, su publicación parcial y/o total en otro medio tiene que ser autorizado por el director de la Revista Visionario Digital.
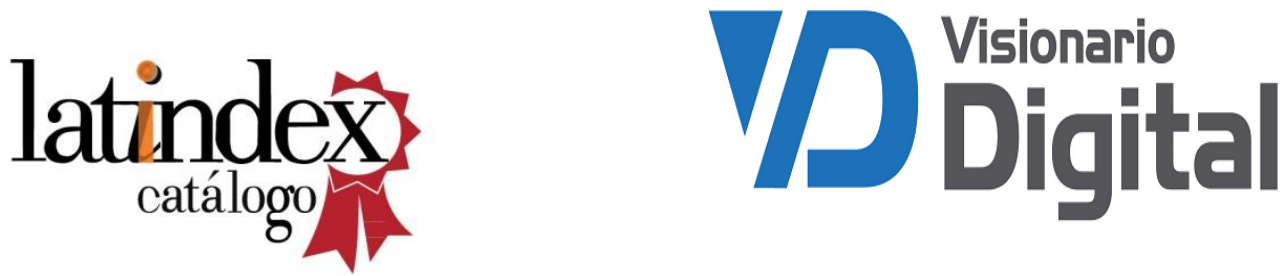